\title{
Antibiotic Resistance, Virulence Factors, Phenotyping, and Genotyping of Non-Escherichia coli Enterobacterales from the Gut Microbiota of Healthy Subjects
}

\author{
Alberto Amaretti ${ }^{1,2,+}{ }^{\circledR}$, Lucia Righini ${ }^{1,+}{ }^{+}$, Francesco Candeliere ${ }^{1}$, Eliana Musmeci ${ }^{1}$, \\ Francesca Bonvicini ${ }^{3}$ (D), Giovanna Angela Gentilomi ${ }^{3,4}$, Maddalena Rossi 1,2 \\ and Stefano Raimondi ${ }^{1, *(D)}$ \\ 1 Department of Life Sciences, University of Modena and Reggio Emilia, via Campi 103, 41125 Modena, Italy; \\ alberto.amaretti@unimore.it (A.A.); lucia.righini@unimore.it (L.R.); francesco.candeliere@unimore.it (F.C.); \\ eliana.musmeci@unimore.it (E.M.); maddalena.rossi@unimore.it (M.R.) \\ 2 Biogest-Siteia, University of Modena and Reggio Emilia, Modena, Viale Amendola 2, \\ 42122 Reggio Emilia, Italy \\ 3 Department of Pharmacy and Biotechnology, Alma Mater Studiorum-University of Bologna, \\ Via Massarenti 9, 40138 Bologna, Italy; francesca.bonvicini4@unibo.it (F.B.); \\ giovanna.gentilomi@unibo.it (G.A.G.) \\ 4 Unit of Microbiology, Alma Mater Studiorum-University of Bologna, S. Orsola-Malpighi Hospital, \\ Via Massarenti 9, 40138 Bologna, Italy \\ * Correspondence: stefano.raimondi@unimore.it; Tel.: +39-059-205-8595 \\ + These authors contributed equally to the work.
}

Received: 2 March 2020; Accepted: 5 March 2020; Published: 7 March 2020

\begin{abstract}
Non-Escherichia coli Enterobacterales (NECE) can colonize the human gut and may present virulence determinants and phenotypes that represent severe heath concerns. Most information is available for virulent NECE strains, isolated from patients with an ongoing infection, while the commensal NECE population of healthy subjects is understudied. In this study, 32 NECE strains were isolated from the feces of 20 healthy adults. 16S rRNA gene sequencing and mass spectrometry attributed the isolates to Klebsiella pneumoniae, Klebsiella oxytoca, Enterobacter cloacae, Enterobacter aerogenes, Enterobacter kobei, Citrobacter freundii, Citrobacter amalonaticus, Cronobacter sp., and Hafnia alvei, Morganella morganii, and Serratia liquefaciens. Multiplex PCR revealed that K. pneumoniae harbored virulence genes for adhesins ( $m r k D$, ycfM, and $k p n)$ and enterobactin (entB) and, in one case, also for yersiniabactin ( $y b t S$, irp1, irp2, and fyuA). Virulence genes were less numerous in the other NECE species. Biofilm formation was spread across all the species, while curli and cellulose were mainly produced by Citrobacter and Enterobacter. Among the most common antibiotics, amoxicillin-clavulanic acid was the sole against which resistance was observed, only Klebsiella strains being susceptible. The NECE inhabiting the intestine of healthy subjects have traits that may pose a health threat, taking into account the possibility of horizontal gene transfer.
\end{abstract}

Keywords: Enterobacterales; Klebsiella; Enterobacter; Citrobacter; virulence; antibiotic resistance; biofilm

\section{Introduction}

Important enteric pathogens belong to Enterobacterales, a bacterial order within the phylum Proteobacteria. This order encompasses permanent colonizers of the human gut that, in healthy conditions, constitute minor bacterial components of the microbiota. Opportunistic Enterobacterales can persist as gut commensals without inducing any infections, as long as the microbiota is balanced and 
the complex and dense bacterial community prevents their overgrowth. A bloom of Enterobacterales may occur as a result of disturbance of the microbiota, yielding pathogen-mediated infections and triggering inflammatory host responses.

Escherichia coli is the most studied among the Enterobacterales with regards to the traits that differentiate commensalism and pathogenicity. It normally colonizes the intestine but comprises both harmless commensals and different pathogenic variants that may instigate infections in the gut or in other tissues [1,2]. Virulent strains of E. coli isolated from infected patients attracted most research interest $[3,4]$, but also fecal isolates from healthy subjects and environmental strains are the target of increasing attention, aiming to determine the pathogenic potential of a wider biodiversity reservoir $[5,6]$.

Many non-E. coli Enterobacterales (hereinafter referred to as NECE) that can colonize the gut (e.g., Klebsiella, Enterobacter, Citrobacter, and Serratia) also present traits that can confer them virulence and pathogenicity or phenotypes that may result in severe heath concern, such as multidrug resistance [7-10]. The greatest efforts have been carried out to describe virulent strains, generally isolated from patients with an ongoing infection, while the pathogenic potential of NECE inhabiting the gut of healthy subjects has not been thoroughly investigated with genetic and phenotypic analysis, except for some genera [9]. The research herein presented aims to fill this gap, providing a genotypic and phenotypic description of the NECE population isolated from the feces of 20 healthy adults, and to complement a previous study that described the E. coli population of the same cohort of subjects [5]. A set of 32 NECE strains was isolated, taxonomically classified, subjected to PFGE genotyping, and described in terms of the genotypic determinants and phenotypic traits that may confer on them potential pathogenicity or invasiveness.

Information on the genes associated to virulence is detailed especially for Klebsiella spp., where a number of genes associated to harmful traits were identified, such as those encoding adhesins, siderophores (e.g., enterobactin, aerobactin, yersiniabactin), protectines, or invasins (responsible for mucoid phenotype and invasiveness), and involved in allantoin metabolism [7,11]. For other NECE genera, such as Enterobacter, Cronobacter, and Citrobacter, the knowledge of the genetic determinants associated to virulence and invasiveness is less comprehensive and, with few exceptions (e.g., Citrobacter koseri), mainly acquired from better characterized pathogens [12-15].

In addition to fimbrial and afimbrial adhesins, the production of surface cellulose structures and curli favors the adhesion of Enterobacterales and can exert a significant role in enteric biofilm-related infections [16,17]. Although not directly involved in pathogenic mechanisms, the acquisition of multiple antibiotic resistances favors the success of opportunistic Enterobacterales pathogens in invasion, survival, and spread, severely complicating the containment and treatment of infections $[9,18]$. Therefore, the occurrence of drug resistant bacteria within a commensal population and the possibility to exchange genetic material by horizontal gene transfer may represent a major health concern.

In the present study, multiplex PCR assays were utilized to screen the NECE, isolated from the feces of healthy subjects, for the presence of 17 main virulence genes associated to those of Klebsiella and E. coli [19-21]. From the point of view of the phenotype, the isolates were characterized for the ability to form biofilm and to yield curli and surface cellulose, were screened for the susceptibility to the most common antibiotics and for the ability to act as recipients in conjugation experiments, and biochemical tests were performed to compare the metabolic profile.

\section{Results}

\subsection{Counting and Isolation of NECE}

The selective differential medium HiCrome Coliform Agar (HCCA) was utilized to count and isolate E. coli [5] and NECE from fecal samples of 20 healthy adults. Total counts in HCCA ranged from $4.6 \times 10^{5}$ to $2.2 \times 10^{8} \mathrm{cfu} / \mathrm{g}$ (Figure 1). Blue colonies attributed to E. coli overcounted the pink ones attributed to NECE in all the samples except V11 (Figure 1; Supplementary Figure S1). NECE ranged between $<10^{4}$ and $1.3 \times 10^{8} \mathrm{cfu} / \mathrm{g}$ and, except in V11, accounted for a minority of total Enterobacterales 
(NECE + E. coli), the 75th percentile being the 5.7\% (Figure 1). In some cases, NECE were not recovered, being outnumbered by E. coli. Spearman's rank correlation analysis excluded any significant correlation between NECE and E. coli counts.

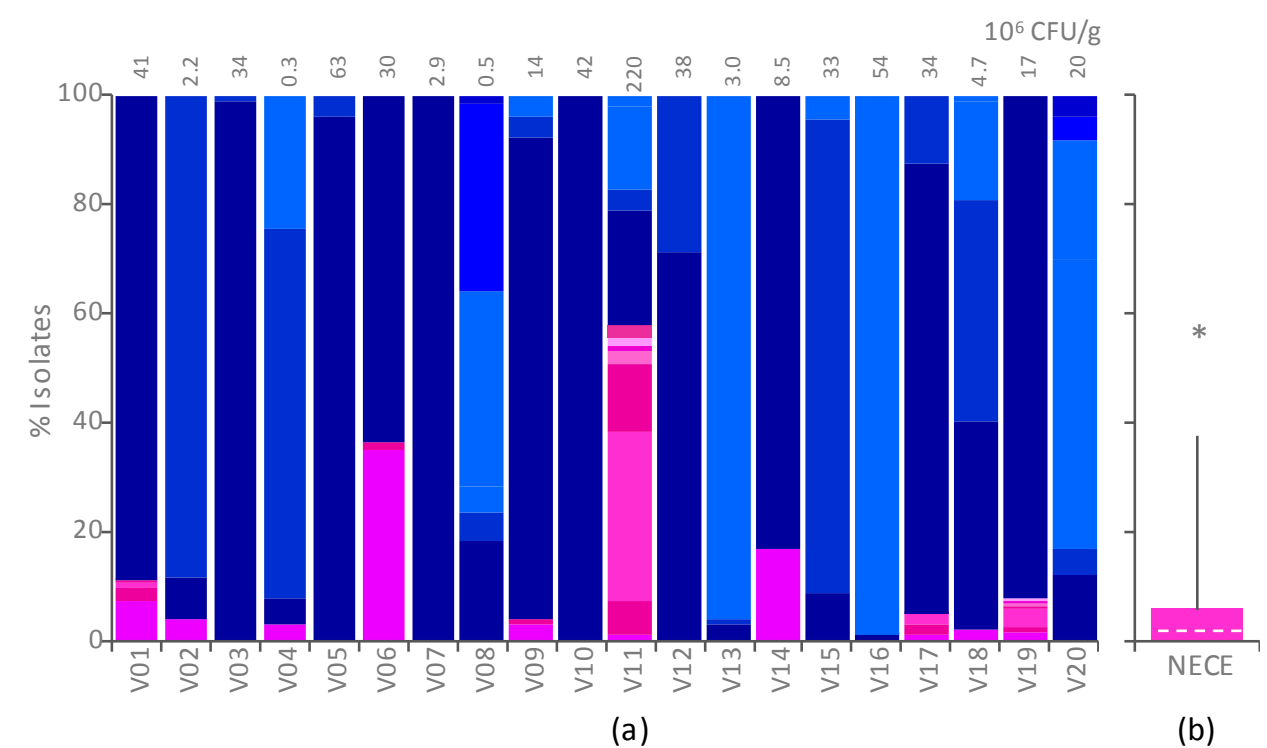

Figure 1. Counts of Escherichia coli and non-Escherichia coli Enterobacterales (NECE), enumerated onto HiCrome Coliform Agar (HCCA) plates. (a) Percentage of colonies attributed to E. coli (blue shades) and NECE (pink shades) in the feces of 20 subjects. For each subject, different shades indicate different biotypes according to enterobacterial repetitive intergenic consensus-PCR (ERIC-PCR) and random amplification of polymorphic DNA-PCR (RAPD-PCR) fingerprinting. The total count of Enterobacterales (E. coli + NECE) is reported in the top margin. (b) Distribution of the percentage of NECE colonies. The median (dashed line), the 25th and 75th percentiles (colored box), the 10th and 90 th percentiles (whiskers), and outliers $\left(^{*}\right)$ are indicated.

\subsection{Taxonomic Attribution and PFGE Genotyping}

The isolates putatively attributed to NECE were clustered in 32 different biotypes utilizing ERIC-PCR (enterobacterial repetitive intergenic consensus-PCR) and RAPD-PCR (random amplification of polymorphic DNA-PCR) fingerprinting. A representative isolate of each biotype was assigned a taxonomic designation utilizing $16 \mathrm{~S}$ rRNA gene sequencing and MALDI-TOF MS (Supplementary Table S1). The genus Klebsiella was the most represented (14/32), with the species K. pneumoniae (10 strains) and Klebsiella oxytoca (4) found in seven and three fecal samples, respectively. The genus Enterobacter was represented by eight strains belonging to Enterobacter cloacae (6), Enterobacter aerogenes, and Enterobacter kobei (1 strain each). Other strains belonged to Citrobacter (4 to Citrobacter freundii and 1 to Citrobacter amalonaticus), Cronobacter sp. (2), and to Hafnia alvei, Morganella morganii, and Serratia liquefaciens (1 strain each).

PFGE highlighted a wide diversity of the NECE isolates, which did not cluster according to the taxonomic attribution (Figure 2). 


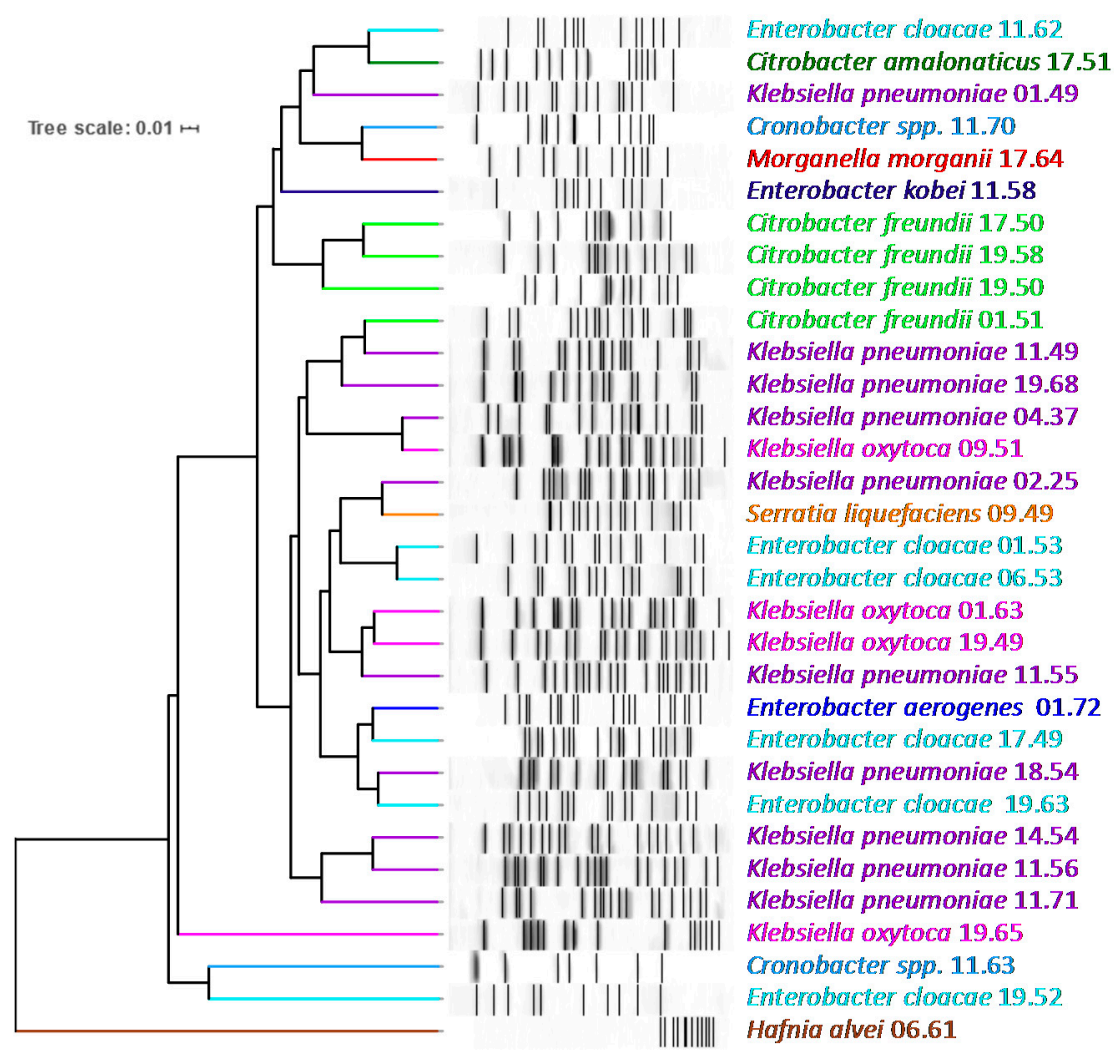

Figure 2. XbaI-PFGE pattern of NECE strains: unweighted pair group method with arithmetic means (UPGMA) dendrogram derived from Dice's coefficients, calculated based on the band profile. Strains are colored based on their MALDI-TOF MS taxonomic attribution.

\subsection{Virulence Genotyping}

PCR was used to investigate 17 virulence genes encoding adhesins (fimH1, $m r k D, k p n$, and ycfM), siderophores (enterobactin, ent $B$; aerobactin, iut $A$; yersiniabactin, irp-1, irp2, ybtS, fyuA; catechols receptor, iroN; and other, $k f u)$, protectines or invasins $(K 2, m a g A, \operatorname{rmp} A$, and $\operatorname{traT})$, and involved in allantoin metabolism (allS).

Most strains of K. pneumoniae harbored the $m r k D, y c f M$, and $k p n$ encoding adhesins and entB encoding enterobactin (Figure 3). Only K. pneumoniae 11.55 was positive to the main virulence genes involved in the synthesis of Yersinia siderophore, including $y b t S$ (encoding for the synthase), irp 1 and irp2 (for regulatory proteins), and fyuA (for the siderophore receptor). irp 2 was also detected in most of the other strains of K. pneumoniae although they lacked the counterpart ybtS. All the K. pneumoniae strains were negative to the genes $K 2, m a g A$, and $r m p A$ associated with hypermucoid phenotype and invasivity, except for K. pneumoniae 01.49 that was positive to K2. Similarly, other virulence genes, such as allS, $k f u$, and iut $A$ occurred only once among the tested strains.

The strains of $K$. oxytoca harbored entB (three out of four isolates) but were negative to most of the other virulence genes. A sole strain harbored $y t b S$. Most of Cronobacter and Enterobacter isolates were characterized by the presence of the gene irp 2 but never harbored $y b t S$ or other Yersinia siderophore genes. A few strains were positive to $m r k D$ or entB.

The strains ascribed to Citrobacter, H. alvei, and M. morganii were negative to those virulence genes whose presence could not be excluded by primer-blast search. The strain of $S$. liquefaciens was positive to $y t b S$. 
C. amalonaticus 17.51

C. freundii 01.51

C. freundii 17.50

C. freundii 19.50

C. freundii 19.58

Cronobacter sp. 11.63

Cronobacter sp. 11.70

E. aerogenes 01.72

E. cloacae 01.53

E. cloacae 06.53

E. cloacae 11.62

E. cloacae 17.49

E. cloacae 19.52

E. cloacae 19.63

E. kobei 11.58

H. alvei 06.61

K. oxytoca 01.63

K. oxytoca 09.51

K. oxytoca 19.49

K. oxytoca 19.65

K. pneumoniae 01.49

K. pneumoniae 02.25

K. pneumoniae 04.37

K. pneumoniae 11.49

K. pneumoniae 11.55

K. pneumoniae 11.56

K. pneumoniae 11.71

K. pneumoniae 14.54

K. pneumoniae 18.54

K. pneumoniae 19.68

M. morganii 17.64

S. liquefaciens 09.49

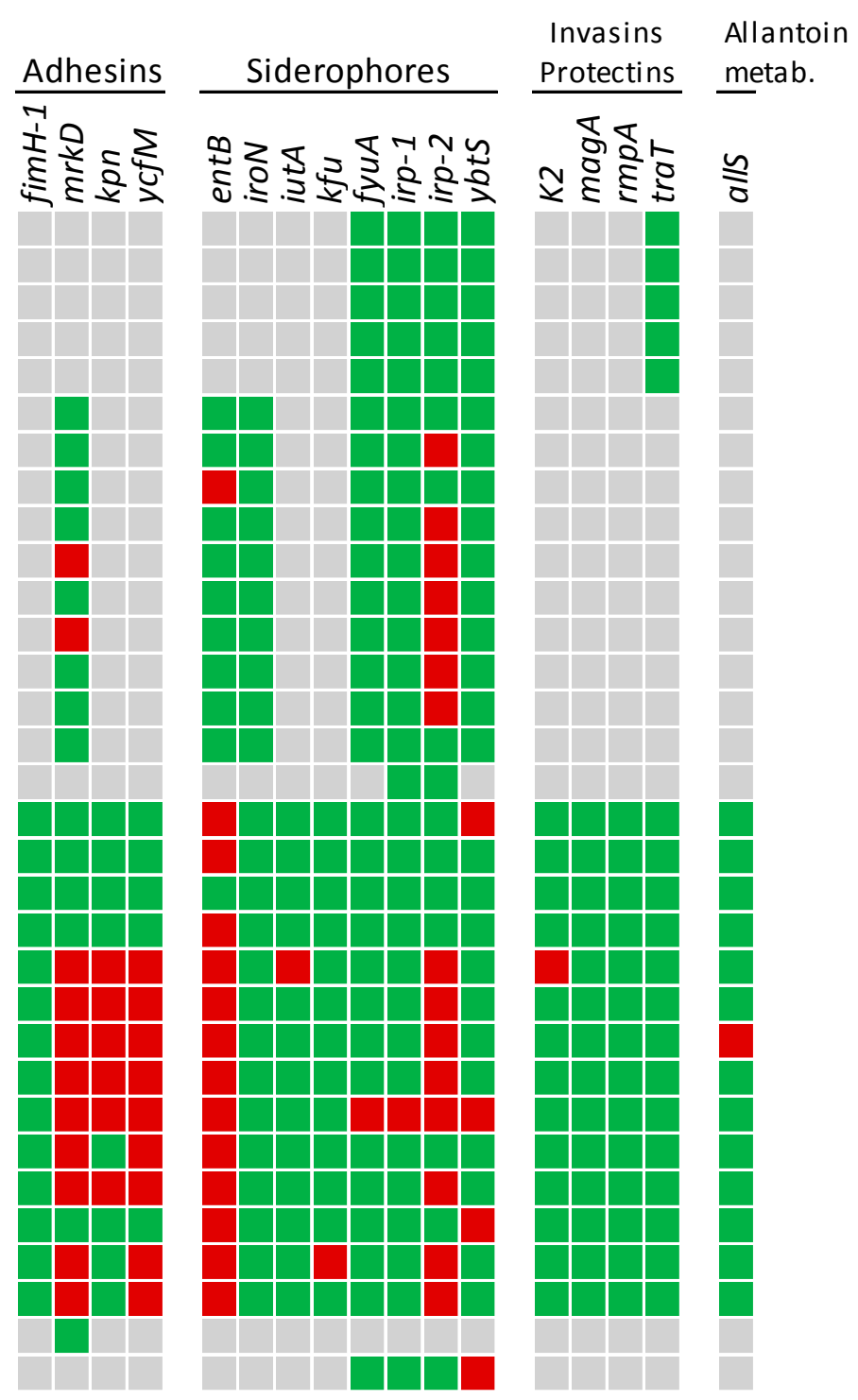

Figure 3. PCR assay of the NECE isolates for the presence of virulence genes. Colors: red, positive amplification; green, negative amplification; grey, PCR analysis not performed since the gene was putatively absent based on a primer-blast search.

\subsection{Biofilm Formation and Production of Curli and Cellulose}

NECE strains were tested for biofilm formation in minimal and rich media (M9 and LBWS, respectively; Supplementary Figure S2). The vast majority of the strains (26 out of 32), belonging to all the species except E. aerogenes, H. alvei, and M. morganii, formed biofilm in M9 (Figure 4; Supplementary Figure S2). Biofilm formation was less frequent in LBWS, being observed only in 10 strains of K. oxytoca, K. pneumoniae, and S. liquefaciens. The extent of biofilm production was always less abundant in the rich medium compared to $\mathrm{M} 9(p<0.05)$.

Extracellular cellulose was detected in most of Citrobacter and Enterobacter strains, in five strains of K. pneumoniae and two out of four of K. oxytoca, in H. alvei and in S. liquefaciens. Curli were produced by nearly all Citrobacter, Cronobacter, and Enterobacter strains and by one isolate belonging to K. oxytoca. The isolates of $H$. alvei and M. morganii were also positive to curli. The strains belonging to Citrobacter, E. cloacae, H. alvei, and K. oxytoca 19.49 produced both cellulose and curli. 


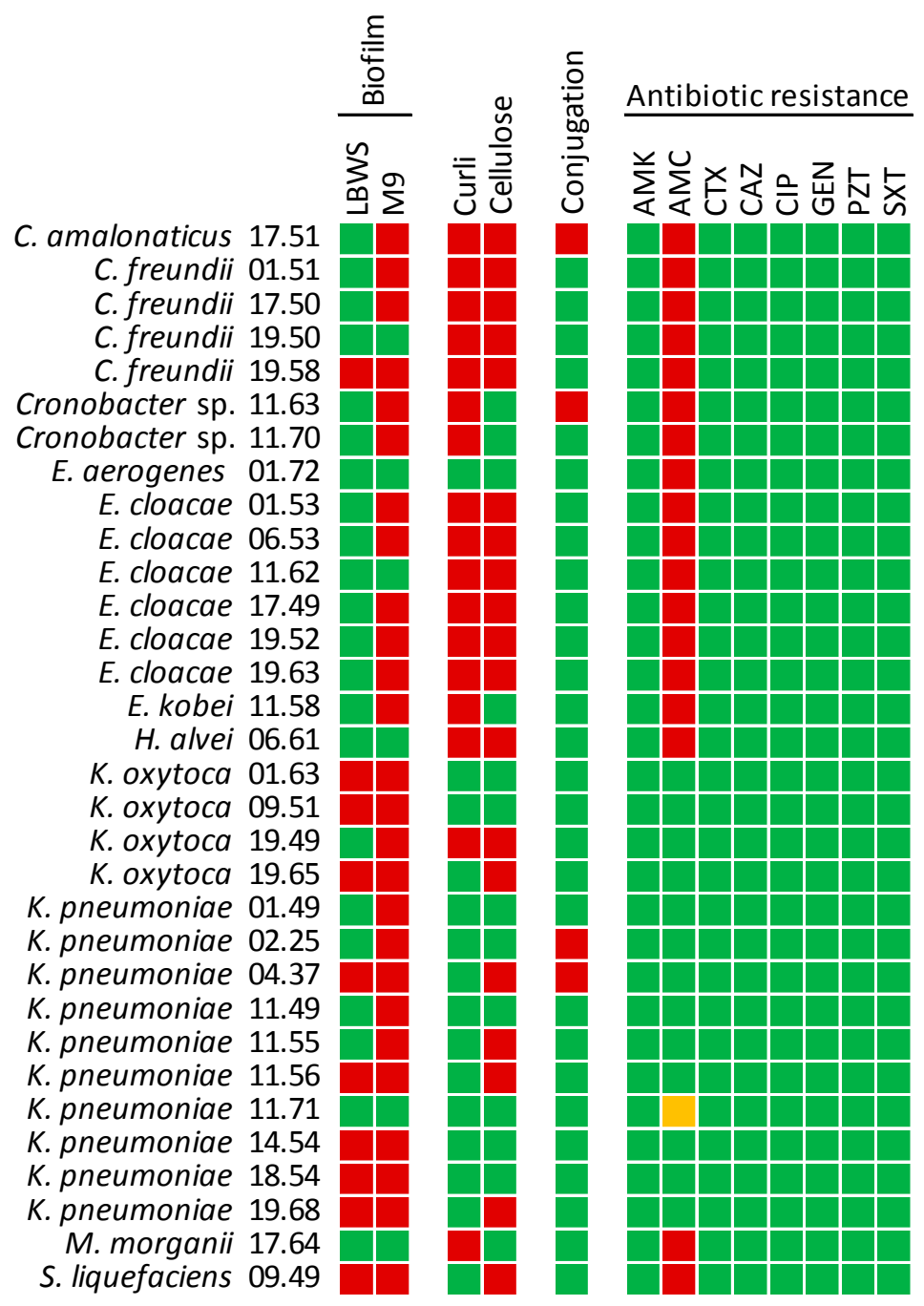

Figure 4. Phenotypic characterization of NECE isolates: biofilm formation in LBWS and M9 media, curli and cellulose production, conjugation, and antibiotic resistance. Colors: red, positive; green, negative. For antibiotics: red, resistant; green, susceptible, yellow, intermediate. Antibiotics: amikacin (AMK), amoxicillin-clavulanic acid (AMC), cefotaxime (CTX), ceftazidime (CAZ), ciprofloxacin (CIP), gentamicin (GEN), piperacillin-tazobactam (PZT), and trimethoprim-sulfamethoxazole (SXT).

\subsection{Conjugation}

The strains were challenged as conjugation recipients for receiving pOX38: Cm plasmid from E. coli N4i. Only two strains of K. pneumoniae, and single strains of Cronobacter and Citrobacter amalonaticus succeeded in plasmid acquisition (Figure 4).

\subsection{Antibiotic Resistance}

Phenotypic susceptibility to amikacin, amoxicillin-clavulanic acid, cefotaxime, ceftazidime, ciprofloxacin, gentamicin, piperacillin-tazobactam, and trimethoprim-sulfamethoxazole was assayed. Amoxicillin-clavulanic acid was the sole antibiotic against which few isolates presented some resistance, with all the strains of Enterobacter, Citrobacter, Cronobacter, H. alvei, M. morganii, and S. liquefaciens being resistant. All the 14 biotypes of Klebsiella spp. were sensitive to the whole set of tested antibiotics, with the exception of K. pneumoniae 11.71 that was partially resistant to amoxicillin-clavulanic acid, presenting a minimum inhibitory concentration (MIC) intermediate between resistance and susceptibility thresholds. 


\subsection{Biochemical Characterization}

The fermentation of substrates and some distinctive enzymatic reactions and metabolic routes were assayed utilizing the API 20 E system (Figure 5). Generally, the NECE strains were positive to $\beta$-galactosidase. Most strains were capable of utilizing citrate, glucose, mannose, inositol, sorbitol, rhamnose, sucrose, melibiose, amygdalin, and arabinose. The main exceptions were $M$. morganii that could utilize only glucose, $H$. alvei that fermented a restricted number of sugars, and some Citrobacter, Cronobacter, and Enterobacter strains that exhibited specific substrate preferences. The majority of the isolates produced either lysine decarboxylase (Klebsiella) or ornithine decarboxylase (Cronobacter and Enterobacter). Urease was characteristic of Klebsiella, while arginine dihydrolase was found in most Enterobacter, Citrobacter, and Cronobacter. Acetoin was produced by Cronobacter, Enterobacter, Hafnia, and Klebsiella. Indole was produced by K. oxytoca and by few other strains, $\mathrm{H}_{2} \mathrm{~S}$ by two strains of Citrobacter freundii. Only S. liquefaciens was positive to gelatinase. All the strains except $M$. morganii and S. liquefaciens exhibited denitrifying activity, in most cases yielding nitrite. Nitrate reduction to $\mathrm{N}_{2}$ was observed in K. pneumoniae and few other strains.

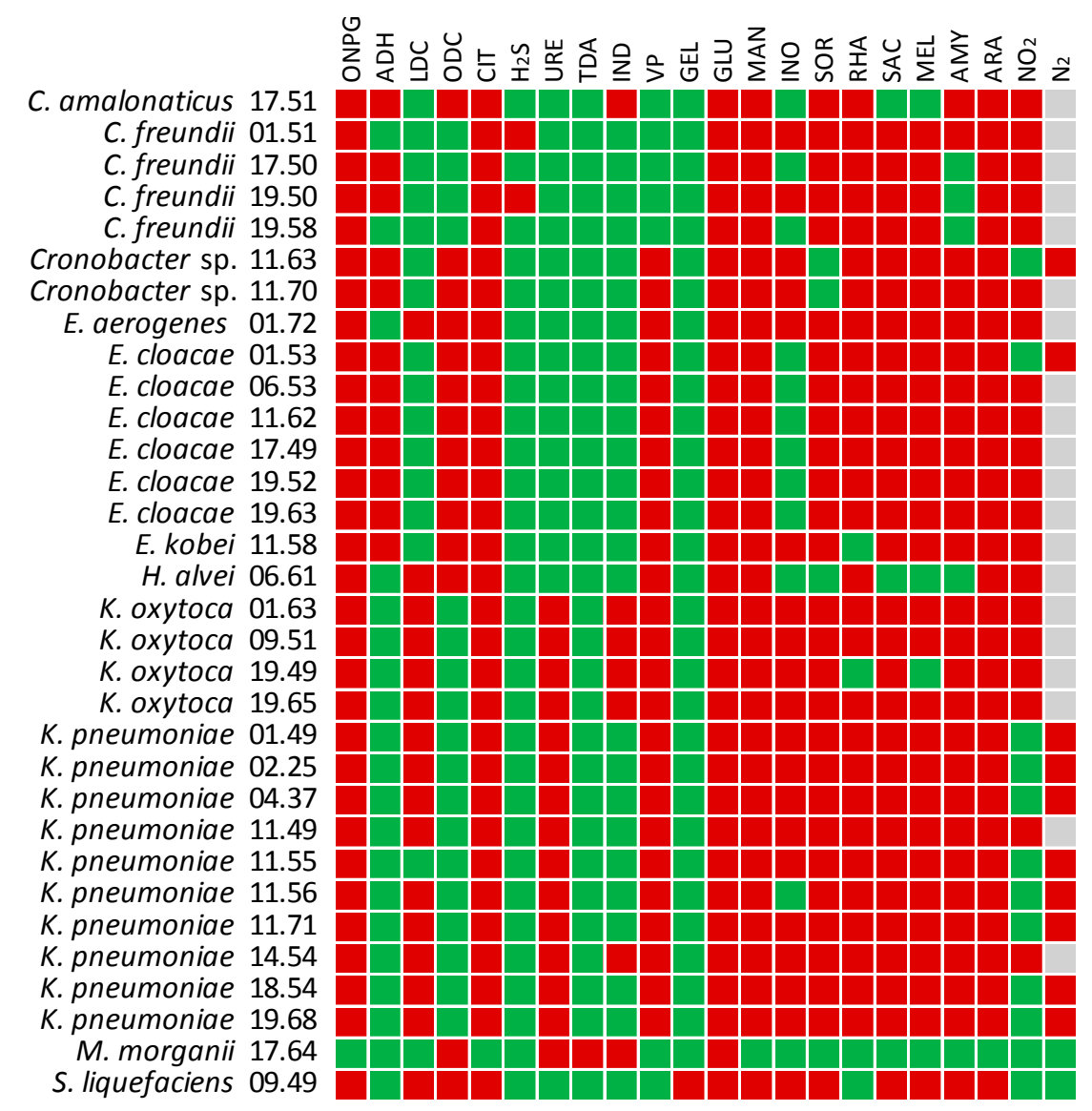

Figure 5. Biochemical reaction profiles of NECE isolates in the API $20 \mathrm{E}$ assay: $\beta$-galactosidase (ONPG), arginine dihydrolase $(\mathrm{ADH})$, lysine decarboxylase (LDC), ornithine decarboxylase (ODC), citrate utilization (CIT), production of hydrogen sulfide $\left(\mathrm{H}_{2} \mathrm{~S}\right)$, urease (URE), tryptophan deaminase (TDA), indole (Kovac's test, IND), acetoin (Voges-Proskauer test, VP), gelatinase (GEL), fermentation of glucose (GLU), mannitol (MAN), inositol (INO), sorbitol (SOR), rhamnose (RHA), sucrose (SAC), melibiose (MEL), amygdalin (AMY), arabinose (ARA), and reduction of nitrates to nitrites $\left(\mathrm{N}_{2} \mathrm{O}\right)$ or nitrogen $\left(\mathrm{N}_{2}\right.$, tested only in case of negative $\mathrm{N}_{2} \mathrm{O}$ ). Colors: red, positive; green, negative. 


\section{Discussion}

Thirty-two NECE strains were isolated from the feces of 20 healthy adults that did not present any dysbiosis, and thus as members of a relatively balanced gut microbiota. The load of Enterobacterales was in the order of millions or tens of millions per gram of feces, with a sole exception where they reached the magnitude of $10^{8}$. NECE represented a small population of Enterobacterales, with E. coli being on average 20 times more abundant, and a minor component of the whole microbiota, being less than $0.1 \%$. The genus Klebsiella, which is ubiquitous in nature, colonizing humans, animals, and plants, and frequently detected in waters, sewages, and soils, was the most represented, encompassing 14 of the 32 strains. K. pneumoniae, the most frequently isolated species (10 strains), was present in 7 out of 20 fecal samples, in accordance to the $35 \%$ of healthy adults from which it was isolated in a pioneering study [22].

K. pneumoniae encompasses opportunistic pathogens that can cause human infections in lungs, urinary tract, and bloodstreams, mostly to hospitalized and/or immunocompromised patients [23,24]. Virulence of K. pneumoniae is associated to the presence of capsule and pili, to the production of lipopolysaccharides and siderophores, to allantoin utilization, and to iron uptake systems, efflux pumps, and type VI secretion systems [7]. Surface molecules, such as capsular polysaccharides and lipopolysaccharides, are some of the major virulence factors that Klebsiella use to protect itself from the host innate immune apparatus. Furthermore, the capability to compete for iron has a pivotal role to the establishment of the infection. The genes involved in iron assimilation are generally clustered in pathogenicity islands, large chromosomal regions that were likely acquired by horizontal transfer. Virulence genetic determinants can be located both in the core or in the accessory genome [7], the former also including metabolic genes required for some species to cause disease.

Among the $10 \mathrm{~K}$. pneumoniae isolates, all but one strain harbored entB, encoding a common catecholate siderophore located in the core genome, and $m r k D$, involved in the synthesis of type 3 pili that promote adherence to the surfaces [19]. The genes encoding other adhesins, such as ycfM and $k p n$ were detected in nine and five strains, respectively. Only two K. pneumoniae strains were positive to the yersiniabactin gene $y b t S$, a common virulence factor associated with human infections $[25,26]$, and one of the two also harbored irp1. These genes are involved in the synthesis of the siderophore yersiniabactin by virulent Yersinia strains, which harbor them within the high-pathogenicity island (HPI). HPI is widely distributed among members of the order Enterobacterales, including E. coli, K. pneumoniae, Citrobacter spp., Salmonella enterica, Serratia liquefaciens, and Enterobacter spp. [27,28]. In addition, irp2, another marker of HPI, was detected in the majority of the strains of K. pneumonia and E. cloacae, albeit they lacked the counterpart ybtS.

Although Enterobacterales are normally present as a low fraction of commensal bacteria in the healthy gut, their numbers can increase in the inflamed gut, and take advantage over other commensals [29]. Siderophores are major contributors of exploitative competition, since iron is an essential nutrient present in very low amounts in the gut and may play a role in virulence. Overall, K. pneumoniae 11.55 was the strain equipped with the broadest set of virulence genes involved in iron metabolism, including those encoding enterobactin (entB), yersiniabactin (irp1, irp2, and ybtS), and, the sole strain among the 32 isolates, also the Yersinia siderophore receptor $(f y u A)$.

In agreement with their commensal behavior, none of the K. pneumoniae strains were positive for the two genes associated with invasive infections, i.e., the mucoviscosity-associated gene mag $A$ and the regulator of mucoid phenotype $\operatorname{rmp} A[30,31]$, that are associated with a hypervirulent and hypermucoid phenotype. In our isolates, the presence of other genetic determinants of virulence was sporadic or quite rare. In the gut of the healthy host, the Enterobacterales resides in the outer loose mucus layer, separated from the epithelial mono-cell barrier by an inner dense mucin coat [32]. During the infection, they penetrate the mucus layer, interact with the epithelial cells, and may breach the mucosal barrier. In case these enterobacteria strains reach other tissues, biofilm formation may act as a fitness factor concurring to pathogenesis [33]. Adhesion factors and extracellular matrix components are involved in formation of biofilms [34]. All but one strain produced biofilm in minimal medium M9, whereas this 
phenotype was less frequent when growth occurred in the rich medium LBWS. The higher extent of biofilm formation in M9 is consistent with the fact that this mineral medium is more challenging for these bacteria. Extracellular cellulose structures, determined in five strains, were consistent with the capability to form biofilm on both media. Curli were found in a sole strain that presented also cellulose structures (19.58 CA) and produced biofilm on both the substrates.

Based on genetic and functional features, some K. pneumoniae isolates present a higher potential to cause infections, albeit they are present at low charge in the microbiota of healthy hosts. The link between colonization and infection by K. pneumoniae in hospitalized patients has been demonstrated, with robust evidence that their own microbiota is the main source of the infective strains $[35,36]$. Thus, potentially more virulent $K$. pneumoniae strains may take advantage of critical conditions, becoming responsible for nosocomial infections [37].

The isolates of $K$. oxytoca, another prominent pathogen that may be involved in diseases and life-threatening infections [38,39], generally encoded the siderophore gene ent $B$, but were negative to most of the other virulence genes. A sole strain harbored the gene encoding the yersiniabactin siderophore. Biofilm production was a general feature of the K. oxytoca strains, regardless of the presence of curli or cellulose structures.

Similar considerations are valid for the other NECE strains herein described, belonging to genera that may have clinical relevance, such as Enterobacter, Citrobacter, and Cronobacter. The isolates were generally capable of forming biofilm and producing curli and cellulose and were negative for most virulence genes. However, unlike Klebsiella that shares many virulence genes with E. coli, the genetic determinants of virulence of these genera have not been fully disclosed.

In this study emerged that NECE isolates from feces of healthy subjects are still quite susceptible to most of the antibiotics. This is important, since any treatment of opportunistic outbreaks of NECE (e.g., in case nosocomial infections) requires antibiotics, but resistance developments would seriously curb the therapeutic options [40]. All the isolates of Klebsiella were sensitive to the whole set of tested antibiotics. Amoxicillin-clavulanic acid was the sole antibiotic against which was detected some resistance: the strains ascribed to the genus Enterobacter and the isolates belonging to Citrobacter, Cronobacter, Hafnia alvei, Morganella morganii, and Serratia liquefaciens were all resistant to this combination of antibiotics. Interestingly, Enterobacterales presented the highest increase in terms of relative abundance in a short-term amoxicillin-clavulanic acid treatment in healthy adults [41]. Some genera belonging to this family, such as Enterobacter and Citrobacter, are recognized as intrinsically resistant [42], and may take advantage to this antibiotic treatment. In general, the profile of resistance was independent by the subject of the fecal sample, but two clusters encompassing sensitive or resistant strains were sharply differentiated by taxonomy.

PFGE genotyping was carried out to evaluate the genetic similarity among the bacterial isolates of this study and highlighted a wide dispersion of the strains, regardless their taxonomic attribution and phylogenetic relationships. The spreading of the strains regardless of species or genera may be attributed to the presence of plasmids, the horizontal acquisition of additional genes from diverse species of Enterobacterales, and to the exchange of mobile elements that rapidly integrate and promote DNA shuffling, in agreement with the capability of some strains to accept DNA by conjugation from E. coli as a donor. However, the biochemical profiling mostly clustered the strains in species (data not shown), confirming that energy production and conservation, and lipid, amino acid, and nucleotide metabolism are part of the conserved reactome, despite the genome plasticity.

The Enterobacterales encountered in this study are generally recognized as opportunistic pathogens, with some potential capability to cause disease, on the basis of predicted virulence factors. Except for K. pneumoniae hypervirulent strains, NECE virulence seems more associated to the host features than to the strain traits. According to the similar results obtained for the E. coli isolated from the same cohort of healthy subjects, the absence of antibiotic resistance for most of the tested antibiotics does not pose a serious challenge for infection control. This highlights the stratification 
of antibiotic resistance distribution among healthy and hospitalized/diseased subjects, with NECE associated risk increasing with both illness and antibiotic therapy.

\section{Materials and Methods}

\subsection{Isolation and Enumeration of Enterobacterales}

Fresh fecal samples were collected from 20 healthy adult subjects who gave written informed consent regarding their participation in the study in accordance with the protocol approved by the local research ethics committee (reference number: 974/2019/SPER-UNIMO-ENTEROPOP; Comitato etico dell'Area Vasta Emilia Nord, Italy). The subjects-10 males and 10 females aged 35 to 45 , following a western omnivore diet, and who had not been treated with prebiotics and/or probiotics for 1 month and antibiotics for 3 months-were enrolled among the employees of the University of Modena and Reggio Emilia and their relatives and were not in relationship with the researchers.

Feces were homogenized $(10 \% w / v)$ in isotonic Buffered Peptone Water (Sigma, Steinheim, Germany), then serial dilutions were spread onto plates of HiCrome Coliform Agar (HCCA, Sigma) and incubated at $37^{\circ} \mathrm{C}$. The medium differentiates E. coli (blue colonies) from NECE (salmon to red). For each subject, up to 48 colonies of putative NECE were picked and clustered into biotypes with ERIC-PCR [43] and RAPD-PCR [44] fingerprint presenting Pearson's similarity $>75 \%$.

\subsection{PFGE Genotyping}

PFGE was performed according to PulseNet protocol (http://www.cdc.gov/pulsenet/PDF/ecolishigella-salmonella-pfge-protocol-508c.pdf). The genomic DNA was digested with $50 \mathrm{U}$ of XbaI at $37^{\circ} \mathrm{C}$ for $3 \mathrm{~h}$. Fragments were resolved in a CHEF-DRIII apparatus (Bio-Rad, Hercules, CA, USA) using counter-clamped homogeneous electric field electrophoresis $(24 \mathrm{~h}$ at $6.0 \mathrm{~V} / \mathrm{cm}$; initial switch time, $2.2 \mathrm{~s}$; final switch time, $54.2 \mathrm{~s}$ ). The run was digitally captured and analyzed with GelCompare II 6.0 software (Applied Maths NV, Sint-Martens-Latem, Belgium). Dice coefficient was computed to evaluate similarity between band profiles (position tolerance, $1 \%$; optimization, $1 \%$ ) and to derive an UPGMA dendrogram (unweighted pair group method with arithmetic means). Strains were ascribed to the same pulsotype if PFGE profile possessed $>85 \%$ similarity.

\subsection{Taxonomic Attribution}

A strain for each biotype was taxonomically characterized by partial sequencing of the $16 \mathrm{~S}$ rRNA gene sequencing, utilizing primers targeting the V1-V3 portion. Primer sequences and PCR conditions were set up according to Raimondi et al. [45]. The sequences, obtained from a service provider (Eurofins Genomics, Ebersberg, Germany), were compared to those in SILVA SSU database utilizing SINA Aligner v1.2.11 (https://www.arb-silva.de/aligner/).

In addition, the MALDI-TOF MS-based biotyping was carried using the MALDI Biotyper 3.1 system (Bruker Daltonics, Bremen, Germany). Sample preparation for MALDI-TOF MS was performed as previously described with minor modifications [46]. Briefly, colonies of fresh overnight culture were placed on a MALDI sample slide (Bruker Daltonics) and dried at room temperature. The sample was then overlaid with $1 \mu \mathrm{L}$ of matrix solution ( $\alpha$-cyano-4-hydroxycinnamic acid in $50 \%$ acetonitrile and $2.5 \%$ trifluoroacetic acid) and dried at room temperature. A MALDI-TOF MS measurement was performed with a Bruker MicroFlex MALDI-TOF MS (Bruker Daltonics) using FlexControl software and a Escherichia coli DH5 $\alpha$ protein extract (Bruker Daltonics) was placed on the calibration spot of the sample slide for external calibration. Spectra collected in the positive-ion mode within a mass range of 2000-20,000 Da were analyzed using a Bruker Biotyper (Bruker Daltonics) automation control and the Bruker Biotyper 3.1 software and library (a database with 5627 entries). High confidence species identification was accepted, if the $\log$ (score) was $\geq 2.00$, low confidence species identification $\log$ (score) values $(\geq 1.70$ and $<2.00$ ) were accepted if the three best matches showed the same species name. Any results with $\log ($ score $)<1.70$ were considered as an unacceptable identification. 


\subsection{Profiling of Virulence Genes}

All the isolates were screened by multiplex-PCR for the genes associated to 17 virulence factors: alls, entB, fimH, fyuA, iroN, irp1, irp2, iutA, K2, kfu, kpn, magA, mrkD, rmpA, traT, ybtS, and ycfM. Primer sequences and amplification conditions were set up according to El Fertas-Aissani et al. [19], Compain et al. [20], and Johnson et al. [21]. In order to assess the possibility to obtain the amplicon in the different NECEs species, a search with the primer-blast tool (https://www.ncbi.nlm.nih.gov/ tools/primer-blast/) was performed for all the set of primers developed for K. pneumoniae and E. coli. The result of PCR amplification was reported only for the species in which the annealing and the possibility to yield an amplicon were predicted.

\subsection{Biofilm and Phenotype Assays}

Biofilm formation was quantified with crystal violet in the microtiter assay described in [47]. Two growth media were compared: Luria Bertani without salt (LBWS) and M9 (BD Difco, Sparks, MD, USA) containing $4 \mathrm{~g} / \mathrm{L}$ glucose and $0.25 \mathrm{~g} / \mathrm{L}$ yeast extract. Strains exhibiting a specific biofilm formation (i.e., the ratio between crystal violet absorbance at $570 \mathrm{~nm}$ and culture turbidity at $600 \mathrm{~nm}$ ) $>1$. The data herein reported are the means of three independent experiments, each carried out in triplicate.

The strains were screened for curli and cellulose production utilizing LBWS agar plates supplemented with the appropriate stain [48]. Red colonies in Congo red-supplemented plates were considered positive to curli. Colonies in calcofluor white-supplemented plates that emitted fluorescence due to UV exposure (315-400 nm) were considered positive to cellulose.

\subsection{Solid Mating Conjugation Experiments}

The strains were screened as recipients in conjugation experiments with the donor E. coli N4i pOX38:Cm (N4i: EcN immE7 Gmr; pOX38:Cm: Tra+ RepFIA+ Cmr) [5]. The donor and the recipient strains were cultured and put in contact onto LB plates under the conditions described in [5]. HCCA and HCCA with $20 \mu \mathrm{g} / \mathrm{mL}$ chloramphenicol were utilized to differentiate recipient, transconjugant, and donor colonies [5].

\subsection{Antibiotic Susceptibility}

The strains were tested for antimicrobial susceptibility with a Vitek2 semi-automated system (bioMerieux, Marcy-l'Étoile, France). Minimum inhibitory concentrations (MICs) were interpreted according to EUCAST (European Committee on Antimicrobial Susceptibility Testing-www.eucast.org) and susceptibility $(\mathrm{S})$ or resistance $(\mathrm{R})$ were defined based on the following thresholds $(\mathrm{mg} / \mathrm{L})$ : amikacin, $\mathrm{S}<8$ and $\mathrm{R}>16$; amoxicillin/clavulanic acid, $\mathrm{S}<8$ and $\mathrm{R}>8$; cefotaxime, $\mathrm{S}<8$ and $\mathrm{R}>2$; ceftazidime, $\mathrm{S}<8$ and $\mathrm{R}>8$; ciprofloxacin, $\mathrm{S}<0.5$ and $\mathrm{R}>1$; gentamicin, $\mathrm{S}<2$ and $\mathrm{R}>4$; piperacillin + tazobactam, $\mathrm{S}<8$ and $\mathrm{R}>16$; trimethoprim/sulfamethoxazole, $\mathrm{S}<40$ and $\mathrm{R}>80$.

\subsection{Biochemical Characterization}

The strains were tested for distinctive enzymatic reactions and metabolic routes utilizing API $20 \mathrm{E}$ test system (bioMerieux, France), according to the manufacturer's instructions.

Supplementary Materials: Supplementary materials can be found at http://www.mdpi.com/1422-0067/21/5/1847/s1.

Author Contributions: Conceptualization, G.A.G., F.B., M.R, and S.R.; data curation, S.R., F.C., and A.A.; investigation, A.A., F.C., L.R., E.M., S.R., and F.B.; methodology, S.R., L.R., F.B., M.R., and G.A.G.; project administration, M.R.; resources, M.R. and G.A.G.; supervision, M.R.; visualization, A.A.; writing-original draft, F.B., S.R., A.A. and M.R.; writing-review \& editing, G.A.G. All authors have read and agreed to the published version of the manuscript.

Funding: Please add: This research received no external funding.

Conflicts of Interest: The authors declare no conflict of interest. 


\section{Abbreviations}

$\begin{array}{ll}\text { NECE } & \text { non-E. coli Enterobacterales } \\ \text { HCCA } & \text { HiCrome Coliform Agar } \\ \text { MALDI-TOF MS } & \text { Matrix-assisted laser desorption ionization-time of flight mass spectrometry } \\ \text { ERIC } & \text { Enterobacterial repetitive intergenic consensus } \\ \text { RAPD } & \text { Random Amplification of Polymorphic DNA } \\ \text { UPGMA } & \text { Unweighted pair group method with arithmetic means }\end{array}$

\section{References}

1. Leimbach, A.; Hacker, J.; Dobrindt, U. E. coli as an all-rounder: The thin line between commensalism and pathogenicity. Curr. Top. Microbiol. Immunol. 2013, 358, 3-32. [CrossRef] [PubMed]

2. Manges, A.R.; Geum, H.M.; Guo, A.; Edens, T.J.; Fibke, C.D.; Pitout, J.D.D. Global extraintestinal pathogenic Escherichia coli (ExPEC) lineages. Clin. Microbiol. Rev. 2019, 32. [CrossRef] [PubMed]

3. Clermont, O.; Christenson, J.K.; Denamur, E.; Gordon, D.M. The Clermont Escherichia coli phylo-typing method revisited: Improvement of specificity and detection of new phylo-groups. Environ. Microbiol. Rep. 2013, 5, 58-65. [CrossRef] [PubMed]

4. Croxen, M.A.; Law, R.J.; Scholz, R.; Keeney, K.M.; Wlodarska, M.; Finlay, B.B. Recent advances in understanding enteric pathogenic Escherichia coli. Clin. Microbiol. Rev. 2013, 26, 822-880. [CrossRef]

5. Raimondi, S.; Righini, L.; Candeliere, F.; Musmeci, E.; Bonvicini, F.; Gentilomi, G.; Starčič Erjavec, M.; Amaretti, A.; Rossi, M. Antibiotic resistance, virulence factors, phenotyping, and genotyping of E. coli isolated from the feces of healthy subjects. Microorganisms 2019, 7, 251. [CrossRef]

6. Sarowska, J.; Futoma-Koloch, B.; Jama-Kmiecik, A.; Frej-Madrzak, M.; Ksiazczyk, M.; Bugla-Ploskonska, G.; Choroszy-Krol, I. Virulence factors, prevalence and potential transmission of extraintestinal pathogenic Escherichia coli isolated from different sources: Recent reports. Gut Pathog. 2019, 11, 10. [CrossRef]

7. Martin, R.M.; Bachman, M.A. Colonization, Infection, and the accessory genome of Klebsiella pneumoniae. Front. Cell. Infect. Microbiol. 2018, 8, 4. [CrossRef]

8. Davin-Regli, A.; Pagès, J.M. Enterobacter aerogenes and Enterobacter cloacae; versatile bacterial pathogens confronting antibiotic treatment. Front. Microbiol. 2015, 6, 392. [CrossRef]

9. Liu, L.; Chen, D.; Liu, L.; Lan, R.; Hao, S.; Jin, W.; Sun, H.; Wang, Y.; Liang, Y.; Xu, J. Genetic diversity, multidrug resistance, and virulence of Citrobacter freundii from diarrheal patients and healthy individuals. Front. Cell. Infect. Microbiol. 2018, 8, 233. [CrossRef]

10. Gajdács, M.; Urbán, E. Resistance Trends and epidemiology of citrobacter-enterobacter-serratia in urinary tract infections of inpatients and outpatients (RECESUTI): A 10-year survey. Medicina (Kaunas) 2019, 55, 285. [CrossRef]

11. Lee, C.R.; Lee, J.H.; Park, K.S.; Jeon, J.H.; Kim, Y.B.; Cha, C.J.; Jeong, B.C.; Lee, S.H. Antimicrobial resistance of hypervirulent Klebsiella pneumoniae: Epidemiology, hypervirulence-associated determinants, and resistance mechanisms. Front. Cell. Infect. Microbiol. 2017, 7, 483. [CrossRef] [PubMed]

12. Azevedo, P.A.A.; Furlan, J.P.R.; Oliveira-Silva, M.; Nakamura-Silva, R.; Gomes, C.N.; Costa, K.R.C.; Stehling, E.G.; Pitondo-Silva, A. Detection of virulence and $\beta$-lactamase encoding genes in Enterobacter aerogenes and Enterobacter cloacae clinical isolates from Brazil. Braz. J. Microbiol. 2018, 49 (Suppl. 1), 224-228. [CrossRef] [PubMed]

13. Mokracka, J.; Koczura, R.; Kaznowski, A. Yersiniabactin and other siderophores produced by clinical isolates of Enterobacter spp. and Citrobacter spp. FEMS Immunol. Med. Microbiol. 2004, 40, 51-55. [CrossRef]

14. Yuan, C.; Yin, Z.; Wang, J.; Qian, C.; Wei, Y.; Zhang, S.; Jiang, L.; Liu, B. Comparative genomic analysis of Citrobacter and key genes essential for the pathogenicity of Citrobacter koseri. Front. Microbiol. 2019, 10, 2774. [CrossRef]

15. Cruz, A.; Xicohtencatl-Cortes, J.; González-Pedrajo, B.; Bobadilla, M.; Eslava, C.; Rosas, I. Virulence traits in Cronobacter species isolated from different sources. Can. J. Microbiol. 2011, 57, 735-744. [CrossRef]

16. Zogaj, X.; Bokranz, W.; Nimtz, M.; Römling, U. Production of cellulose and curli fimbriae by members of the family Enterobacteriaceae isolated from the human gastrointestinal tract. Infect. Immun. 2003, 71, 4151-4158. [CrossRef] 
17. Tursi, S.A.; Tükel, Ç. Curli-containing enteric biofilms inside and out: Matrix composition, immune recognition, and disease implications. Microbiol. Mol. Biol. Rev. 2018, 82. [CrossRef]

18. Partridge, S.R. Resistance mechanisms in Enterobacteriaceae. Pathology 2015, 47, 276-284. [CrossRef]

19. El Fertas-Aissani, R.; Messai, Y.; Alouache, S.; Bakour, R. Virulence profiles and antibiotic susceptibility patterns of Klebsiella pneumoniae strains isolated from different clinical specimens. Pathol. Biol. (Paris) 2013, 61, 209-216. [CrossRef]

20. Compain, F.; Babosan, A.; Brisse, S.; Genel, N.; Audo, J.; Ailloud, F.; Kassis-Chikhani, N.; Arlet, G.; Decré, D. Multiplex PCR for detection of seven virulence factors and K1/K2 capsular serotypes of Klebsiella pneumoniae. J. Clin. Microbiol. 2014, 52, 4377-4380. [CrossRef]

21. Johnson, J.R.; Porter, S.; Johnston, B.; Kuskowski, M.A.; Spurbeck, R.R.; Mobley, H.L.; Williamson, D.A. Host characteristics and bacterial traits predict experimental virulence for Escherichia coli bloodstream isolates from patients with urosepsis. Open Forum Infect. Dis. 2015, 2. [CrossRef] [PubMed]

22. Davis, T.J.; Matsen, J.M. Prevalence and characteristics of Klebsiella species: Relation to association with a hospital environment. J. Infect. Dis. 1974, 130, 402-405. [CrossRef] [PubMed]

23. Lau, H.Y.; Huffnagle, G.B.; Moore, T.A. Host and microbiota factors that control Klebsiella pneumoniae mucosal colonization in mice. Microbes Infect. 2008, 10, 1283-1290. [CrossRef] [PubMed]

24. Magill, S.S.; Edwards, J.R.; Bamberg, W.; Beldavs, Z.G.; Dumyati, G.; Kainer, M.A.; Lynfield, R.; Maloney, M.; McAllister-Hollod, L.; Nadle, J.; et al. Multistate point-prevalence survey of health care-associated infections. N. Engl. J. Med. 2014, 370, 1198-1208. [CrossRef]

25. Lan, Y.; Zhou, M.; Jian, Z.; Yan, Q.; Wang, S.; Liu, W. Prevalence of pks gene cluster and characteristics of Klebsiella pneumoniae-induced bloodstream infections. J. Clin. Lab. Anal. 2019, 33, e22838. [CrossRef]

26. Lawlor, M.S.; O'connor, C.; Miller, V.L. Yersiniabactin is a virulence factor for Klebsiella pneumoniae during pulmonary infection. Infect. Immun. 2007, 75, 1463-1472. [CrossRef]

27. Schubert, S.; Cuenca, S.; Fischer, D.; Heesemann, J. High-pathogenicity island of Yersinia pestis in Enterobacteriaceae isolated from blood cultures and urine samples: Prevalence and functional expression. J. Infect. Dis. 2000, 182, 1268-1271. [CrossRef]

28. Schubert, S.; Rakin, A.; Heesemann, J. The Yersinia high-pathogenicity island (HPI): Evolutionary and functional aspects. Int. J. Med. Microbiol. 2004, 294, 83-94. [CrossRef]

29. Raffatellu, M. Learning from bacterial competition in the host to develop antimicrobials. Nat. Med. 2018, 24, 1097-1103. [CrossRef]

30. Fang, C.T.; Chuang, Y.P.; Shun, C.T.; Chang, S.C.; Wang, J.T. A novel virulence gene in Klebsiella pneumoniae strains causing primary liver abscess and septic metastatic complications. J. Exp. Med. 2004, 199, 697-705. [CrossRef]

31. Yu, W.L.; Ko, W.C.; Cheng, K.C.; Lee, H.C.; Ke, D.S.; Lee, C.C.; Fung, C.P.; Chuang, Y.C. Association between rmpA and magA genes and clinical syndromes caused by Klebsiella pneumoniae in Taiwan. Clin. Infect. Dis. 2006, 42, 1351-1358. [CrossRef] [PubMed]

32. Johansson, M.E.; Sjovall, H.; Hansson, G.C. The gastrointestinal mucus system in health and disease. Nat. Rev. Gastroenterol. Hepatol. 2013, 10, 352-361. [CrossRef] [PubMed]

33. Flores-Mireles, A.L.; Walker, J.N.; Caparon, M.; Hultgren, S.J. Urinary tract infections: Epidemiology, mechanisms of infection and treatment options. Nat. Rev. Microbiol. 2015, 13, 269-284. [CrossRef] [PubMed]

34. Hobley, L.; Harkins, C.; MacPhee, C.E.; Stanley-Wall, N.R. Giving structure to the biofilm matrix: An overview of individual strategies and emerging common themes. FEMS Microbiol. Rev. 2015, 39, 649-669. [CrossRef] [PubMed]

35. Martin, R.M.; Cao, J.; Brisse, S.; Passet, V.; Wu, W.; Zhao, L.; Malani, P.N.; Rao, K.; Bachman, M.A. Molecular epidemiology of colonizing and infecting isolates of Klebsiella pneumoniae. mSphere 2016, 1. [CrossRef] [PubMed]

36. Gorrie, C.L.; Mirceta, M.; Wick, R.R.; Edwards, D.J.; Thomson, N.R.; Strugnell, R.A.; Pratt, N.F.; Garlick, J.S.; Watson, K.M.; Pilcher, D.V.; et al. Gastrointestinal carriage is a major reservoir of Klebsiella pneumoniae infection in intensive care patients. Clin. Infect. Dis. 2017, 65, 208-215. [CrossRef] [PubMed]

37. Gajdács, M.; Bátori, Z.; Ábrók, M.; Lázár, A.; Burián, K. Characterization of resistance in gram-negative urinary isolates using existing and novel indicators of clinical relevance: A 10-year data analysis. Life (Basel) 2020, 10, 16. [CrossRef] 
38. Carrie, C.; Walewski, V.; Levy, C.; Alexandre, C.; Baleine, J.; Charreton, C.; Coche-Monier, B.; Caeymaex, L.; Lageix, F.; Lorrot, M.; et al. Klebsiella pneumoniae and Klebsiella oxytoca meningitis in infants. Epidemiological and clinical features. Arch. Pediatr. 2019, 26, 12-15. [CrossRef]

39. Pötgens, S.A.; Brossel, H.; Sboarina, M.; Catry, E.; Cani, P.D.; Neyrinck, A.M.; Delzenne, N.M.; Bindels, L.B. Klebsiella oxytoca expands in cancer cachexia and acts as a gut pathobiont contributing to intestinal dysfunction. Sci. Rep. 2018, 8, 12321. [CrossRef]

40. Gajdács, M.; Ábrók, M.; Lázár, A.; Burián, K. Comparative epidemiology and resistance trends of common urinary pathogens in a tertiary-care hospital: A 10-year surveillance study. Medicina (Kaunas) 2019, 55, 356. [CrossRef]

41. MacPherson, C.W.; Mathieu, O.; Tremblay, J.; Champagne, J.; Nantel, A.; Girard, S.A.; Tompkins, T.A. Gut bacterial microbiota and its resistome rapidly recover to basal state levels after short-term amoxicillin-clavulanic acid treatment in healthy adults. Sci. Rep. 2018, 8, 11192. [CrossRef] [PubMed]

42. Leclercq, R.; Cantón, R.; Brown, D.F.; Giske, C.G.; Heisig, P.; MacGowan, A.P.; Mouton, J.W.; Nordmann, P.; Rodloff, A.C.; Rossolini, G.M.; et al. EUCAST expert rules in antimicrobial susceptibility testing. Clin. Microbiol. Infect. 2013, 19, 141-160. [CrossRef] [PubMed]

43. Wilson, L.A.; Sharp, P.M. Enterobacterial repetitive intergenic consensus (ERIC) sequences in Escherichia coli: Evolution and implications for ERIC-PCR. Mol. Biol. Evol. 2006, 23, 1156-1168. [CrossRef] [PubMed]

44. Quartieri, A.; Simone, M.; Gozzoli, C.; Popovic, M.; D’Auria, G.; Amaretti, A.; Raimondi, S.; Rossi, M. Comparison of culture-dependent and independent approaches to characterize fecal bifidobacteria and lactobacilli. Anaerobe 2016, 38, 130-137. [CrossRef]

45. Raimondi, S.; Luciani, R.; Sirangelo, T.M.; Amaretti, A.; Leonardi, A.; Ulrici, A.; Foca, G.; D'Auria, G.; Moya, A.; Zuliani, V.; et al. Microbiota of sliced cooked ham packaged in modified atmosphere throughout the shelf life: Microbiota of sliced cooked ham in MAP. Int. J. Food Microbiol. 2019, 16, 200-208. [CrossRef]

46. Mencacci, A.; Monari, C.; Leli, C.; Merlini, L.; De Carolis, E.; Vella, A.; Cacioni, M.; Buzi, S.; Nardelli, E.; Bistoni, F.; et al. Typing of nosocomial outbreaks of Acinetobacter baumannii by use of matrix-assisted laser desorption ionization-time of flight mass spectrometry. J. Clin. Microbiol. 2013, 51, 603-606. [CrossRef]

47. Sicard, J.F.; Vogeleer, P.; Le Bihan, G.; Rodriguez Olivera, Y.; Beaudry, F.; Jacques, M.; Harel, J. $\mathrm{N}$-Acetyl-glucosamine influences the biofilm formation of Escherichia coli. Gut Pathog. 2018, 22, 10-26. [CrossRef]

48. Vogeleer, P.; Tremblay, Y.D.N.; Jubelin, G.; Jacques, M.; Harel, J. Biofilm-forming abilities of shiga toxin-producing Escherichia coli isolates associated with human infections. Appl. Environ. Microbiol. 2015, 28, 1448-1458. [CrossRef] 